\title{
COMPRESSIVE IMAGING OF SUBWAVELENGTH STRUCTURES
}

\author{
ALBERT C. FANNJIANG
}

\begin{abstract}
The problem of imaging extended targets (sources or scatterers) is formulated in the framework of compressed sensing with emphasis on subwavelength resolution.

The proposed formulation of the problems of inverse source/scattering is essentially exact and leads to the random partial Fourier measurement matrix. In the case of square-integrable targets, the proposed sampling scheme in the Littlewood-Paley wavelet basis block-diagonalizes the scattering matrix with each block in the form of random partial Fourier matrix corresponding to each dyadic scale of the target.

The resolution issue is analyzed from two perspectives: stability and the signal-to-noise ratio (SNR). The subwavelength modes are shown to be typically unstable. The stability in the subwavelength modes requires additional techniques such as near-field measurement or illumination. The number of the stable modes typically increases as the negative $d$-th (the dimension of the target) power of the distance between the target and the sensors/source. The resolution limit is shown to be inversely proportional to the SNR in the high SNR limit.

Numerical simulations are provided to validate the theoretical predictions.
\end{abstract}

\section{INTRODUCTION}

The Compressed Sensing paradigm is supplying a fresh perspective on the imaging problems, including source inversion and inverse scattering (see the extensive literature in [8]) and we aim to analyze these classical problems from the perspective of compressed sensing.

The main purposes of the paper are two folds: (i) to formulate the problems of inverse source and scattering for extended targets in the framework of compressed sensing and analyze it by the fundamental results on the random Fourier measurements $[4,5,6,7,21,22]$ and (ii) to use the compressed sensing solution thus obtained as a starting point to investigate the problem of subwavelength resolution in the presence of noise, in particular, to discuss the idea of extracting subwavelength information by near-field measurement and illumination, common in nano-optics $[20]$.

The important feature of our formulation of the imaging problems is that it is essentially exact (without the paraxial approximation as used in [18]) and is for extended (periodic or non-periodic) targets. As such, our formulation provides an instructive example for assessing the power of the compressed sensing techniques when applied to the physical problems of imaging. In the case of inverse scattering for square-integrable targets, we use the Littlewood-Paley wavelet basis and propose a sampling scheme by which the scattering matrix can be block-diagonalized with each block in the form of random partial Fourier matrix corresponding to each dyadic scale present in the target structure. By this approach we can image the extended target scale-by-scale.

We analyze the resolution issue from two perspectives. First we define the stably recoverable (or stable for short) modes in terms of the noise amplification factor. We show that the subwavelength modes are typically unstable. To achieve stability in the subwavelength reconstruction we need additional techniques such as near-field measurement or illumination with which the number of the

The research is partially supported by the NSF grant DMS - 0908535 . 
stable modes typically increases as the negative $d$-th (the dimension of the target) power of the distance between the target and the sensors/source. Second, we take into account the signal-to-noise ratio (SNR) in the resolution analysis and show that the resolution limit is inversely proportional to the SNR in the high SNR limit.

The paper is organized as follows. We treat the case of periodic targets using the Fourier basis, first for the inverse scattering in Section 2 and then for the inverse (Born) scattering problem in Section 3. We present numerical results confirming the theoretical predictions in Section 4. In Section 5 we analyze the case of square-integrable targets using the Littlewood-Paley basis and point out the extension to three dimensions. We conclude in Section 6.

\section{SOURCE INVERSION}

First we consider imaging of an extended source (the target) in two dimensional $(z, x)$-plane. We assume that the target is located in the line $z=z_{0}$.

The target is represented by the variable source amplitude $\sigma(x)$ which is assumed to be periodic with period $L$ and admits the Fourier expansion

$$
\sigma(x)=\sum_{k=-\infty}^{\infty} \hat{\sigma}_{k} e^{i 2 \pi k x / L} .
$$

We consider the class of band-limited functions with $\hat{\sigma}_{k}=0$ except for

$$
k \in[-(m-1) / 2,(m-1) / 2]
$$

in (1) where $m$ is an odd integer so that there are at most $m$ relevant Fourier modes.

The wave propagation in the free space is governed by the Helmholtz equation

$$
\Delta u+\omega^{2} u=0 \text {. }
$$

where $\omega=2 \pi / \lambda$ is the frequency assuming the wave speed is unity. In two dimensions, the Green function $G(\mathbf{r})$ is

$$
G(\mathbf{r})=\frac{i}{4} H_{0}^{(1)}(\omega|\mathbf{r}|), \quad \mathbf{r}=(z, x)
$$

where $H_{0}^{(1)}$ is the zeroth order Hankel function of the first kind. $G$ can be expressed by the Sommerfeld integral representation

$$
G(\mathbf{r})=\frac{i}{4 \pi} \int e^{i \omega(|z| \beta(\alpha)+x \alpha)} \frac{d \alpha}{\beta(\alpha)},
$$

where

$$
\beta(\alpha)= \begin{cases}\sqrt{1-\alpha^{2}}, & |\alpha|<1 \\ i \sqrt{\alpha^{2}-1}, & |\alpha|>1\end{cases}
$$

[1]. The integrand in (3) with real-valued $\beta$ (i.e. $|\alpha|<1$ ) corresponds to the homogeneous wave and that with imaginary-valued $\beta$ (i.e. $|\alpha|>1$ ) corresponds to the evanescent (inhomogeneous) wave which has an exponential-decay factor $e^{-\omega|z| \sqrt{\alpha-1}}$.

The signal arriving at the sensor located at $(0, x)$ is given by

$$
\int G\left(z_{0}, x-x^{\prime}\right) \sigma\left(x^{\prime}\right) d x^{\prime}=\frac{i}{2 \omega} \sum_{k} \frac{\hat{\sigma}_{k}}{\beta_{k}} e^{i \omega z_{0} \beta_{k}} e^{i \omega \alpha_{k} x}
$$

by (3) where

$$
\alpha_{k}=\frac{k \lambda}{L}, \quad \beta_{2}=\beta\left(\alpha_{k}\right) .
$$


By (6), the subwavelength structure of the target is encoded in $\hat{\sigma}_{k}$ with $|k| \lambda>L$ which are conveyed by the evanescent waves.

Let $\left(0, x_{j}\right), x_{j}=\xi_{j} L, j=1, \ldots, n$ be the coordinates of the $n$ sensors in the line $z=0$. To set the problem in the framework of compressed sensing we define the signals received by the $n$ sensors as the measurement vector $Y$ and set the target vector $X=\left(X_{k}\right) \in \mathbb{C}^{m}$ as

$$
X_{k}=\frac{i \sqrt{n} e^{i \omega z_{0} \beta_{k}}}{2 \omega \beta_{k}} \hat{\sigma}_{k} .
$$

To avoid a vanishing denominator in (7), we must set $L / \lambda \notin \mathbb{N}$ so that $\beta_{k} \neq 0$. This leads to the form

$$
Y=\Phi X
$$

where the sensing matrix $\boldsymbol{\Phi}=\left[\Phi_{j k}\right] \in \mathbb{C}^{n \times m}$ has the entries

$$
\Phi_{j k}=\frac{1}{\sqrt{n}} e^{i \omega \alpha_{k} x_{j}}=\frac{1}{\sqrt{n}} e^{i 2 \pi k \xi_{j}}, \quad j=1, \ldots, n, \quad k=1, \ldots, m .
$$

Hence the imaging procedure is split into two stages: the first is to "invert" $\Phi$ and solve for $X$, and the second is to deduce $\sigma$ from $X$. The sensing matrix (9) is designed to mimic the random partial Fourier matrix which has the restricted isometry property and is amenable to the compressed sensing techniques (see below). Note that the formulation is essentially exact and no far-field approximation is made.

The main thrust of compressive sensing is that under suitable conditions the inversion can be achieved as the $\ell^{1}$-minimization

$$
\min \|X\|_{1}, \quad \text { subject to } Y=\boldsymbol{\Phi} X
$$

which also goes by the name of Basis Pursuit (BP) and can be solved by linear programming. BP was first introduced empirically in seismology by Claerbout and Muir and later studied mathematically by Donoho and others $[2,9,13]$.

A fundamental notion in compressed sensing under which BP yields the unique exact solution is the restrictive isometry property due to Candès and Tao [6]. Precisely, let the sparsity $s$ of the target vector be the number of nonzero components of $X$ and define the restricted isometry constant $\delta_{s}$ to be the smallest positive number such that the inequality

$$
\left(1-\delta_{s}\right)\|Z\|_{2}^{2} \leq\|\mathbf{\Phi} Z\|_{2}^{2} \leq\left(1+\delta_{s}\right)\|Z\|_{2}^{2}
$$

holds for all $Z \in \mathbb{C}^{m}$ of sparsity at most $s$.

For the target vector $X$ let $X_{s}$ denote the best $s$-sparse approximation of $X$ in the sense of $L^{1}$-norm, i.e.

$$
X^{s}=\operatorname{argmin}\|Z-X\|_{1}, \quad \text { s.t. }\|Z\|_{0} \leq s
$$

where $\|Z\|_{0}$ denotes the number of nonzero components, called the sparsity, of $Z$. Clearly, $X^{s}$ consists of the $s$ largest components of $X$.

Now we state the fundamental result of the RIP approach [3] which is an improvement of the results of $[4,6]$.

Theorem 1. [3] Suppose the restricted isometry constant of $\mathbf{\Phi}$ satisfies the inequality

$$
\begin{gathered}
\delta_{2 s}<\sqrt{2}-1 \\
3
\end{gathered}
$$


Then the solution $X_{*}$ by BP satisfies

$$
\begin{aligned}
\left\|X_{*}-X\right\|_{1} & \leq C_{0}\left\|X-X^{s}\right\|_{1} \\
\left\|X_{*}-X\right\|_{2} & \leq C_{0} s^{-1 / 2}\left\|X-X^{s}\right\|_{1}
\end{aligned}
$$

for some constant $C_{0}$. In particular, if $X$ is s-sparse, then the recovery is exact.

Remark 1. Greedy algorithms have significantly lower computational complexity than linear programming and have provable performance under various conditions. For example under the condition $\delta_{3 s}<0.06$ the Subspace Pursuit $(S P)$ algorithm is guaranteed to exactly recover $X$ via a finite number of iterations [14]. See [19] for a closely related algorithm (CoSaMP).

Theorem 2. [21] Let $\xi_{j}, j=1, \ldots, n$ be independent, uniform random variables in $[0,1]$. Suppose

$$
\frac{n}{\ln n} \geq C \delta^{-2} r \ln ^{2} r \ln m \ln \frac{1}{\eta}, \quad \eta \in(0,1)
$$

for a given sparsity $r$ where $C$ is an absolute constant. Then the restricted isometry constant of the matrix for random Fourier measurements satisfies

$$
\delta_{r} \leq \delta
$$

with probability at least $1-\eta$.

See $[4,7,22]$ for the case when $\xi_{l}$ belong to the discrete subset of $[0,1]$ of equal spacing $1 / \sqrt{m}$.

As a consequence of Theorem 1 and 2, the target vector $X$ can be determined by BP and then the source amplitude $\sigma(x)$ can be reconstructed exactly from (7), including all the subwavelength structures. In other words, in the absence of noise there is essentially no limitation to the resolving power of the compressed sensing technique, subwavelength or not, as long as sufficient number of measurements are made.

When noise is present, however, the performance of the above approach may be severely limited, especially in the recoverability of subwavelength information. Consider the standard model of additive noise

$$
Y^{\varepsilon}=\boldsymbol{\Phi} X+E
$$

where $\|E\|_{2}=\varepsilon>0$ is the size of the noise and the associated relaxation scheme

$$
\min \|X\|_{1}, \quad \text { subject to }\left\|Y^{\varepsilon}-\mathbf{\Phi} X\right\|_{2} \leq \epsilon .
$$

The next result is a restatement of the result of [3] after applying Theorem 2 with $r=2 s, \delta<\sqrt{2}-1$.

Theorem 3. Let $X^{\varepsilon}$ be the solution to (15). Then under the assumptions of Theorem (2) we have

$$
\left\|X^{\varepsilon}-X\right\|_{2} \leq C_{0} s^{-1 / 2}\left\|X-X^{s}\right\|_{1}+C_{1} \varepsilon
$$

with probability at least $1-\eta$ where $C_{0}$ and $C_{1}$ are constants.

See also $[12,23]$ for stability result under the condition of incoherence.

Inverting the relationship (7) with small error in the target vector $X$ produces a mildly amplified error for those $\hat{\sigma}_{k}$ such that

$$
\left|e^{i \omega z_{0} \beta_{k}}\right| \geq e^{-2 \pi}
$$

but significantly amplified error otherwise. Here the transition is not clear-cut, however. The choice of the noise amplification threshold $e^{2 \pi}$ as the stability criterion is convenient but arbitrary; any constant less than one will serve our purpose. 
The stable (or stably recoverable) modes are those corresponding to $\left|\alpha_{k}\right| \leq 1$ as well as $\left|\alpha_{k}\right|>1$ such that

$$
\omega\left|\beta_{k}\right| z_{0} \leq 2 \pi
$$

or equivalently

$$
k \leq L \sqrt{\lambda^{-2}+z_{0}^{-2}}
$$

Hence reducing the distance $z_{0}$ between the sensor array and the target can effectively enlarge the number of stable modes and more of the subwavelength modes become stably recoverable as $z_{0}$ decreases below wavelength. This is the idea behind the near-field imaging systems such as the scanning microscopy.

The above reconstruction essentially consists of two stages: Stage 1 involves the compressed sensing techniques which is always stable (Theorem 3) and Stage 2 is a simple inversion of the diagonal matrix

$$
\operatorname{diag}\left(\frac{i \sqrt{n} e^{i \omega z_{0} \beta_{k}}}{2 \omega \beta_{k}}\right),
$$

cf. (7), which is stable if and only if (18) holds for all $k$ such that $\hat{\sigma}_{k} \neq 0$. When (18) is indeed violated, brute force inversion of the matrix (20) would lead to an enormous error and hence a regularization is called for. In such a case, we can use the well-known Tikhonov regularization to invert (20).

The analysis from (17) to (19) focuses on the stability issue based on the noise amplification factor. On the other hand, it is well accepted that the resolution of any imaging system should be a function of the signal-to-noise-ratio (see [17] and references therein). Let us now analyze the resolution from this perspective.

Let $X^{\varepsilon}=\left(X_{k}^{\varepsilon}\right)$ be the solution of the convex relaxation scheme (15) and let

$$
\hat{\sigma}_{k}^{\varepsilon}=\frac{2 \omega \beta_{k}}{i \sqrt{n}} e^{-i \omega z_{0} \beta_{k}} X_{k}^{\varepsilon}
$$

For simplicity, let us assume that the sparsity of $X$ is at most $s$ and therefore by Theorem 3 we have

$$
\left\|X^{\varepsilon}-X\right\|_{2} \leq C_{1} \varepsilon
$$

where the constant $C_{1}$ is well-behaved as demonstrated in the numerical results of Section 4 .

We say that the Fourier mode $\hat{\sigma}_{k}$ is resolved if the following inequality holds:

$$
\left|\frac{\hat{\sigma}_{k}^{\varepsilon}}{\hat{\sigma}_{k}}-1\right| \leq \frac{1}{K}
$$

where the constant $K$ is sufficiently larger than unity. Using (22) and (21) we obtain

$$
\begin{aligned}
\left|\frac{\hat{\sigma}_{k}^{\varepsilon}}{\hat{\sigma}_{k}}-1\right| & \leq \frac{2 \omega\left|\beta_{k}\right|}{\sqrt{n}}\left|e^{-i \omega z_{0} \beta_{k}}\right| \frac{\left|X_{k}^{\varepsilon}-X_{k}\right|}{\left|\hat{\sigma}_{k}\right|} \\
& \leq 2 C_{1} \omega\left|\beta_{k}\right| \frac{\varepsilon}{\left|e^{i \omega z_{0} \beta_{k}}\right|\left|\hat{\sigma}_{k}\right| \sqrt{n}} .
\end{aligned}
$$

The expression

$$
\mathrm{SNR}_{k}=\left|e^{i \omega z_{0} \beta_{k}}\right|\left|\hat{\sigma}_{k}\right| \sqrt{n} / \varepsilon
$$

has the meaning of the signal-to-noise ratio for the $k$-th Fourier mode and we shall call it as such. 
Assuming that the right hand side of $(23)$ is at most $1 / K$ we obtain from (23) the inequality

$$
\left|\beta_{k}\right| \leq \frac{\mathrm{SNR}_{k}}{2 C_{1} K \omega}
$$

and consequently

$$
\frac{L}{k} \geq\left(\frac{1}{\lambda^{2}}+\left(\frac{\mathrm{SNR}_{k}}{4 \pi C_{1} K}\right)^{2}\right)^{-1 / 2} .
$$

The resolution limit is then obtained by minimizing the left hand side of (25). For simplicity, considering the case that $\mathrm{SNR}_{k}=\mathrm{SNR}$ is independent of $k$ for all nonzero modes $\hat{\sigma}_{k}$ we obtain the resolution limit

$$
\left(\frac{1}{\lambda^{2}}+\left(\frac{\mathrm{SNR}}{4 \pi C_{1} K}\right)^{2}\right)^{-1 / 2}
$$

which essentially says that the resolution is inversely proportional to SNR in the high SNR limit.

Next let us discuss a different mechanism of superresolution available in the context of inverse scattering.

\section{INVERSE SCATTERING}

Consider a periodic scatterer with scattering amplitude $\sigma$ admitting the representation (1). For simplicity, we use the Born scattering model [1] under which the scattered field at $z=0$ is given by

$$
u^{\mathrm{s}}(0, x)=\int G\left(z_{0}, x-x^{\prime}\right) \sigma\left(x^{\prime}\right) u^{\mathrm{i}}\left(z_{0}, x^{\prime}\right) d x^{\prime}
$$

where $u^{\mathrm{i}}(z, x)$ is the incident field. Now with the normally incident plane wave $u^{\mathrm{i}}(z, x)=e^{i \omega z}$, eq. (27) is essentially reduced to (5).

Consider the obliquely incident plane wave $u^{\mathrm{i}}(z, x)=e^{i \omega\left(\alpha x+\beta\left|z-z_{1}\right|\right)}$ where $\beta$ is related to $\alpha$ as in (6) and $z_{1}$, assumed larger than $z_{0}$. Set

$$
\alpha=\frac{q \lambda}{L}, \quad q \in \mathbb{R} .
$$

In the standard setting the illumination field is a homogeneous wave with $|\alpha|<1$. The evanescent illumination $|\alpha|>1$ will also be considered here. In such case, $z_{1}$ is the $z$-coordinate of the illumination source.

The same calculation as before now leads to

$$
u^{\mathrm{S}}(0, x)=\frac{i}{2 \omega} \sum_{k} \frac{\hat{\sigma}_{k}}{\beta_{k}} e^{i \omega z_{0} \beta_{k}} e^{i \omega\left(z_{1}-z_{0}\right) \beta} e^{i \omega \alpha_{k} x}
$$

where instead of (6) we have

$$
\alpha_{k}=\frac{(k+q) \lambda}{L}, \quad \beta_{k}=\left\{\begin{array}{ll}
\sqrt{1-\alpha_{k}^{2}}, & \left|\alpha_{k}\right|<1 \\
i \sqrt{\alpha_{k}^{2}-1}, & \left|\alpha_{k}\right|>1
\end{array} .\right.
$$

Define the target vector $X^{(q)}=\left(X_{k}^{(q)}\right) \in \mathbb{C}^{m}$ as

$$
X_{k}^{(q)}=\frac{i \sqrt{n} e^{i \omega z_{0} \beta_{k}} e^{i \omega\left(z_{1}-z_{0}\right) \beta}}{2 \omega \beta_{k}} \hat{\sigma}_{k}
$$


and proceed as before. Note that $e^{i \omega\left(z_{1}-z_{0}\right) \beta}$ is a constant factor determined solely by the illumination source (distance and angle). To avoid a vanishing denominator we require

$$
\frac{(k+q) \lambda}{L} \neq 1, \quad \forall k .
$$

Theorem 1 and 2 are applicable to the shifted Fourier matrix $\Phi^{(q)}=\left[\Phi_{j k}^{(q)}\right]$ with

$$
\Phi_{j k}^{(q)}=\frac{1}{\sqrt{n}} e^{i 2 \pi(k+q) \xi_{j}}
$$

where $\xi_{j}$ are independent, uniform random variables in $[0,1]$.

The same stability analysis as in Section 2 implies that the stable modes in the Born scattering satisfy the constraint

$$
\left|\alpha_{k}\right|=\frac{|k+q| \lambda}{L} \leq \sqrt{1+\frac{\lambda^{2}}{z_{0}^{2}}}
$$

which implies that for each $q$

$$
-q-\sqrt{\frac{L^{2}}{\lambda^{2}}+\frac{L^{2}}{z_{0}^{2}}} \leq k \leq-q+\sqrt{\frac{L^{2}}{\lambda^{2}}+\frac{L^{2}}{z_{0}^{2}}} .
$$

Let us maximize the range of stable modes (31) under the constraint $\left|e^{i \omega\left(z_{1}-z_{0}\right) \beta}\right|>e^{-2 \pi}$ by considering two different illumination sources: homogeneous and evanescent wave sources. If the target is illuminated by incident homogeneous waves with $|q|<L / \lambda$, then the stable modes are

$$
|k| \leq \frac{L}{\lambda}+\sqrt{\frac{L^{2}}{\lambda^{2}}+\frac{L^{2}}{z_{0}^{2}}} .
$$

The same resolution analysis as before leads to

$$
\left|\frac{\hat{\sigma}_{k}^{\varepsilon}}{\hat{\sigma}_{k}}-1\right| \leq \frac{2 \omega\left|\beta_{k}\right|}{\sqrt{n}}\left|e^{-i \omega z_{0} \beta_{k}}\right|\left|e^{-i \omega\left(z_{1}-z_{0}\right) \beta}\right| C_{1} \frac{\varepsilon}{\left|\hat{\sigma}_{k}\right|} .
$$

The appropriate definition of the signal-to-noise ratio in this case is

$$
\mathrm{SNR}_{k}=\sqrt{n}\left|\hat{\sigma}_{k}\right|\left|e^{i \omega z_{0} \beta_{k}}\right|\left|e^{i \omega\left(z_{1}-z_{0}\right) \beta}\right| / \varepsilon
$$

after taking into account the intensity of illumination. Setting the right hand side of (33) to be at most $1 / K$ then leads to (24) with $\alpha_{k}$ given by (30). which in turn yields the resolution limit

$$
\left(\frac{1}{\lambda}+\sqrt{\frac{1}{\lambda^{2}}+\left(\frac{\mathrm{SNR}}{4 \pi C_{1} K}\right)^{2}}\right)^{-1} .
$$

On the other hand, if the incident wave is evanescent and subject to the constraint $\left|e^{i \omega\left(z_{1}-z_{0}\right) \beta}\right|>$ $e^{-2 \pi}$, which implies

$$
|q|<\sqrt{\frac{L^{2}}{\lambda^{2}}+\frac{L^{2}}{\left(z_{1}-z_{0}\right)^{2}}},
$$

then the stable modes according to (31) satisfy

$$
|k|<\sqrt{\frac{L^{2}}{\lambda^{2}}+\frac{L^{2}}{\left(z_{1}-z_{0}\right)^{2}}}+\sqrt{\frac{L^{2}}{\lambda^{2}}+\frac{L^{2}}{z_{0}^{2}}} .
$$



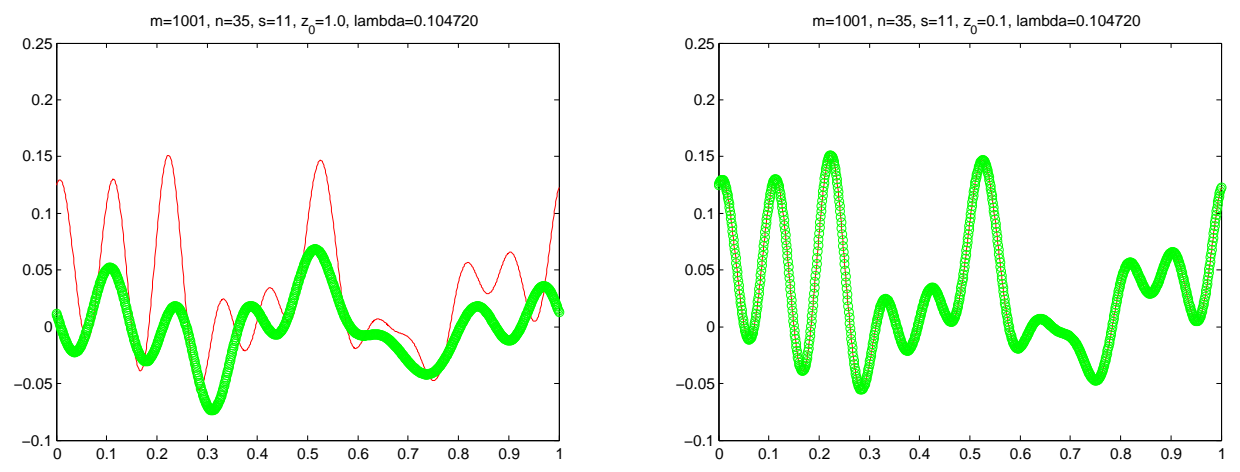

FiguRE 1. In source inversion with far-field measurement (left, $z_{0}=1, n=35$ ), the two subwavelength modes $k= \pm 11$ cause significant errors. With near-field measurement (right, $z_{0}=0.1, n=35$ ) the reconstruction is nearly perfect for target with 20 subwavelength modes. The thin-red curve is the original profile and the thick-green curve is the reconstructed profile. The two curves coincide in the plot on the right.

To maximize the range of stable modes limited by (36) we choose $q_{*}>0$, which is sufficiently close to the right hand side of (35) and satisfies (29), and illuminate the target by a few evanescent waves with $q \in\left[-q_{*}, q_{*}\right]$. The union of their respective stable modes (31) is the totality of stable modes. In optics, the evanescent illumination can be produced physically by, for example, the total internal reflection [20].

Using the same evanescent illumination procedure we can achieve the resolution limit

$$
\left(\sqrt{\frac{1}{\lambda^{2}}+\frac{1}{\left(z_{1}-z_{0}\right)^{2}}}+\sqrt{\frac{1}{\lambda^{2}}+\left(\frac{\mathrm{SNR}}{4 \pi C_{1} K}\right)^{2}}\right)^{-1}
$$

which indicates two ways of improving resolution without increasing the probe frequency: reducing the distance between the plane-wave source and the target, and increasing the signal-to-noise ratio.

\section{Numerical RESUlts}

Numerical methods for convex optimization (such as the Matlab program cvx designed by M. Grant and S. Boyd) are not exact; they compute their results to within a predefined numerical precision or tolerance which is acceptable for most applications. However, for our calculations involving subwavelength modes, the numerical error tends to be amplified enormously and hence severely spoils the reconstruction.

On the other hand, because of the simplicity of the greedy algorithms, the numerical error can be much reduced and the quality of recovery much improved. Of course, the sparsity constraints of the greedy algorithms tend to be more severe than that of the convex relaxation. Since the sparsity constraint is not the main focus of our study, we use the Subspace Pursuit in the numerical calculations.

In our numerical simulations, $L=1, \lambda=\pi / 30, m=1001$ so the subwavelength mode cutoff is at about $|k|=10$. The large $m$ is selected to demonstrate the compressed sensing step.

The numerical results for source inversion are shown in Figure 1. First we image a periodic source with 11 modes, including one subwavelength mode $k=11$ using far-field measurement 

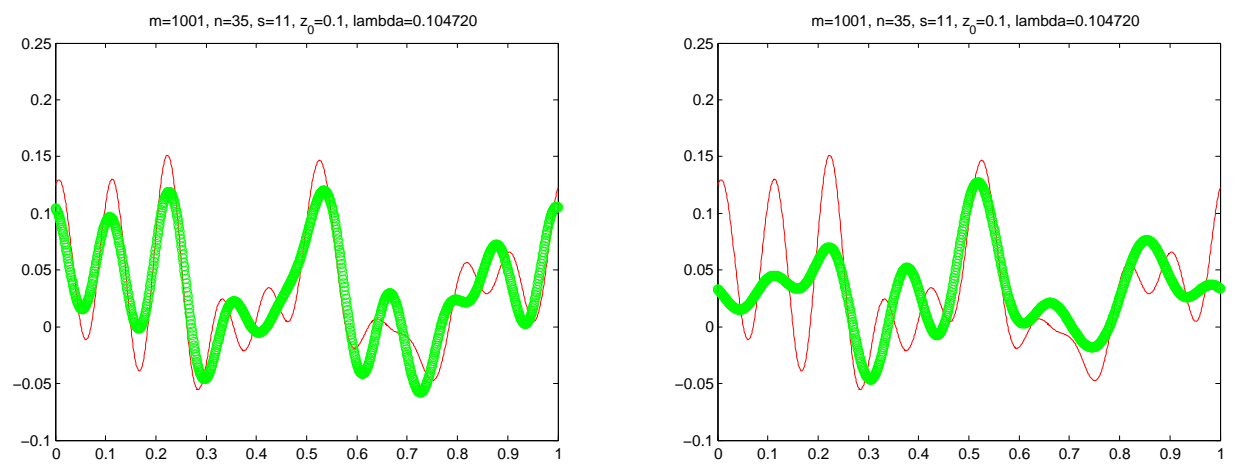

FIGURE 2. In source inversion with near-field measurement with added random noise (left 1\%, right 5\%). The thin-red curve is the original profile and the thickgreen curve is the reconstructed profile.
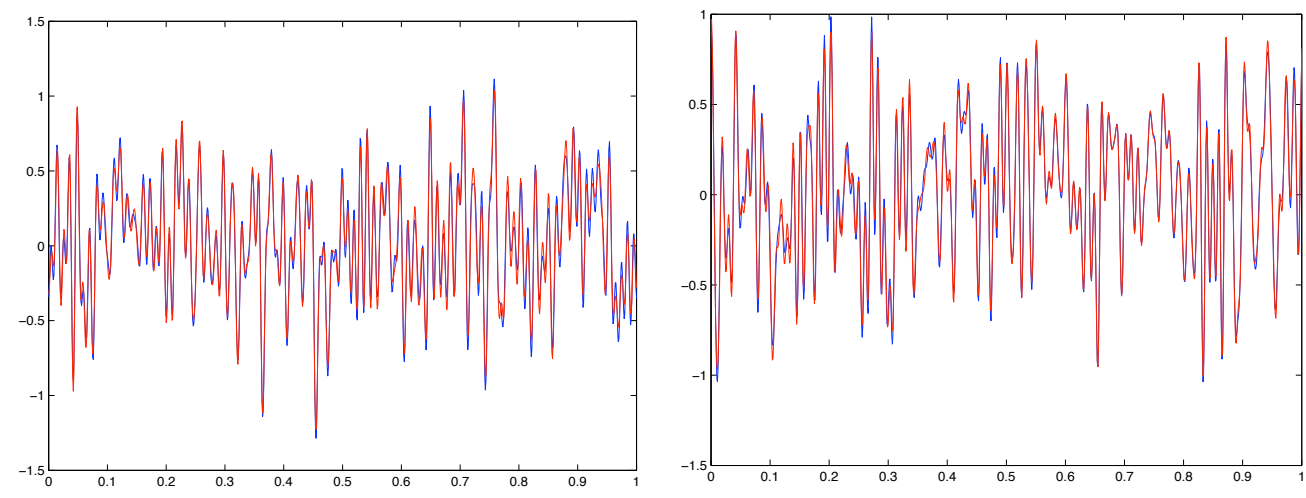

FiguRE 3. Two independent runs (left with $n=70$ right with $n=82$ ) with far-field measurement $\left(z_{0}=1\right)$ and near-field illumination $\left(z_{1}-z_{0}=0.01\right)$. The red curve is the original profile and the blue curve is the reconstructed profile. Except for a few spots, they coincide with each other.

$\left(z_{0}=1, n=35\right)$. The main source of noise in this simulation is the roundoff error. As evident in the left plot of Figure 1 the recovery is not accurate. Indeed, the subwavelength mode is entirely missing in the reconstruction. Reducing the distance between the target and the sensors $\left(z_{0}=0.1\right)$ enables accurate reconstruction (right plot in Figure 1). When additional random noise is added, the quality of recovery with near-field measurement deteriorates in proportion to the amount of noise (Figure 2). With about 5\% random noise, the imaging result with near-field measurement (Figure 2, right) is comparable to that of far-field measurement without added noise (Figure 1, left).

Figure 3 shows the results for inverse Born scattering by the far-field measurement $\left(z_{0}=1\right)$ and the near-field illumination $\left(z_{1}-z_{0}=0.01\right)$ without additional noise. In this case it suffices to run the procedure for two incident modes with $q= \pm 90$.

\section{Extension AND GENERALIZATIONS}


5.1. Non-periodic targets. Let us consider a non-periodic target represented by a square-integrable function $\sigma$.

To represent $\sigma$ we use the Littlewood-Paley basis

$$
\hat{\psi}(\xi)=\left\{\begin{array}{cc}
(2 \pi)^{-1 / 2} . & \pi \leq|\xi| \leq 2 \pi \\
0, & \text { otherwise }
\end{array}\right.
$$

or

$$
\psi(x)=(\pi x)^{-1}(\sin (2 \pi x)-\sin (\pi x)) .
$$

Then the following set of functions

$$
\psi_{p, q}(x)=2^{-p / 2} \psi\left(2^{-p} x-q\right), \quad p, q \in \mathbb{Z}
$$

forms an orthonormal wavelet basis in $L^{2}(\mathbb{R})$ [11]. Expanding the target profile $\sigma(x)$ in the Littlewood-Paley basis $\left\{\psi_{p, q}\right\}$ we have

$$
\sigma(x)=\sum_{p, q \in \mathbb{Z}} \sigma_{p, q} \psi_{p, q}(x)
$$

The scattered field has the far-field asymptotic

$$
u^{\mathrm{S}}(\mathbf{r})=\frac{e^{i \omega|\mathbf{r}|}}{|\mathbf{r}|^{1 / 2}}\left(A(\hat{\mathbf{r}}, \mathbf{d}, \omega)+\mathcal{O}\left(|\mathbf{r}|^{-1}\right)\right), \quad \hat{\mathbf{r}}=\mathbf{r} /|\mathbf{r}|
$$

where the scattering amplitude $A$ (at frequency $\omega$ and the sampling direction $\hat{\mathbf{r}}$ with the incident field $\left.u^{\mathrm{i}}=e^{i \mathbf{d} \cdot \mathbf{r}}\right)$ can be calculated according to

$$
A(\hat{\mathbf{r}}, \mathbf{d}, \omega)=\frac{\omega^{2}}{4 \pi} \int d \mathbf{r}^{\prime} \sigma\left(\mathbf{r}^{\prime}\right) e^{i \omega \mathbf{d} \cdot \mathbf{r}^{\prime}} e^{-i \omega \mathbf{r}^{\prime} \cdot \hat{\mathbf{r}}}, \quad \mathbf{d}=(\alpha, \beta), \quad \alpha^{2}+\beta^{2}=1
$$

[10].

In inverse scattering theory, the scattering amplitudes are measured in the directions $\hat{\mathbf{r}}_{k}=$ $\left(\alpha_{k}, \beta_{k}\right)=\left(\cos \theta_{k}, \sin \theta_{k}\right), k=1, \ldots n$ and denoted by $Y=\left(Y_{k}\right) \in \mathbb{C}^{n}$. By (43) and (41) we have

$$
Y_{k}=\frac{\omega^{2}}{2 \sqrt{2 \pi}} \sum_{p, q \in \mathbb{Z}} 2^{p / 2} \sigma_{p, q} e^{i \omega\left(\alpha-\alpha_{k}\right) 2^{p} q} \hat{\psi}\left(\omega\left(\alpha-\alpha_{k}\right) 2^{p}\right), \quad k=1, \ldots, n .
$$

Let

$$
\begin{aligned}
l=\sum_{j=-p_{*}}^{p-1}\left(2 m_{j}+1\right)+q, \quad|q| \leq m_{p}, \quad|p| \leq p_{*}, \\
k=\sum_{j=-p_{*}}^{p^{\prime}-1}\left(2 n_{j}+1\right)+q^{\prime}, \quad\left|q^{\prime}\right| \leq n_{p}, \quad\left|p^{\prime}\right| \leq p_{*}
\end{aligned}
$$

for some $m_{p}, n_{p}, p_{*} \in \mathbb{N}$ be the column and row indices of the sensing matrix, respectively. Set $m=\sum_{|p| \leq p_{*}}\left(2 m_{p}+1\right)$ and $n=\sum_{|p| \leq p_{*}}\left(2 n_{p}+1\right)$.

Suppose that

$$
\omega 2^{-p_{*}} \geq 2 \pi
$$

and consider, for simplicity, the normal incident field $\alpha=0$. Let $\zeta_{p^{\prime}, q^{\prime}}$ be independent, uniform random variables on $[-1,1]$ and let

$$
\alpha_{k}=\frac{\pi}{\omega 2^{p^{\prime}}} \cdot\left\{\begin{array}{c}
1+\zeta_{p^{\prime}, q^{\prime}}, \quad \zeta_{p^{\prime}, q^{\prime}} \in[0,1] \\
-1+\zeta_{p^{\prime}, q^{\prime}}, \quad \zeta_{p^{\prime}, q^{\prime}} \in[-1,0]
\end{array}\right.
$$


which lies in $[-1,1]$ by the assumption (45). Let the sensing matrix elements be

$$
\Phi_{k, l}=\frac{1}{\sqrt{2 n_{p}+1}} \hat{\psi}\left(\omega \alpha_{k} 2^{p}\right) e^{-i \omega \alpha_{k} 2^{p} q} .
$$

We claim that $\Phi_{k, l}=0$ for $p \neq p^{\prime}$. This is evident from the following calculation

$$
\omega \alpha_{k} 2^{p}=\pi 2^{p-p^{\prime}} \cdot\left\{\begin{array}{cc}
1+\zeta_{p^{\prime}, q^{\prime}}, & \zeta_{p^{\prime}, q^{\prime}} \in[0,1] \\
-1+\zeta_{p^{\prime}, q^{\prime}}, & \zeta_{p^{\prime}, q^{\prime}} \in[-1,0]
\end{array}\right.
$$

whose right hand side is outside the support of $\hat{\psi}$ for $p \neq p^{\prime}$.

For every $p=p^{\prime}$,

$$
\Phi_{k, l}=\frac{1}{\sqrt{2 n_{p}+1}} e^{-i \pi \zeta_{p^{\prime}, q^{\prime}} q}, \quad\left|q^{\prime}\right| \leq n_{p}, \quad|q| \leq m_{p}
$$

which constitute the random partial Fourier matrix. In other words, under the assumption (45) the sensing matrix $\boldsymbol{\Phi}=\left[\Phi_{k, l}\right] \in \mathbb{C}^{n \times m}$ is block-diagonal with each block (indexed by $p$ ) in the form (49).

Let $X=\left(X_{l}\right)$ be the target vector with

$$
X_{l}=\frac{\omega^{2}}{2 \sqrt{2 \pi}} \sqrt{\left(2 n_{p}+1\right) 2^{p}} \sigma_{p, q}, \quad l=\sum_{j=-p_{*}}^{p-1}\left(2 m_{j}+1\right)+q .
$$

We can then express the measurement vector $Y=\left(Y_{k}\right) \in \mathbb{C}^{n}$ as in (8).

Each block represented by the submatrix (49) can be treated as in the periodic case. For each $p,|p| \leq p_{*}$, let $s_{p}$ be the sparsity of the target vector for the scale $2^{p}$ and suppose that the frequency $\omega_{p}$ used to probe the scale $2^{p}$ satisfies

$$
\omega_{p} 2^{p} \geq 2 \pi
$$

Under these assumptions, Theorem 2 yields the sufficient condition

$$
\frac{2 n_{p}+1}{\ln \left(2 n_{p}+1\right)} \geq C \delta^{-2} s_{p} \ln ^{2} s_{p} \ln m \ln \frac{1}{\eta}, \quad \eta \in(0,1)
$$

for the RIP

$$
\delta_{s_{p}} \leq \delta
$$

to hold with probability at least $1-\eta$.

The assumption (50) means that the wavelength is at least as small as the scale being probed. Therefore, this imaging method does not possess the subwavelength resolving power.

Figure 4 shows the result of reconstruction with the Littlewood-Paley basis and the following parameters: $p_{*}=2, m_{p}=100, \forall p ;$ for $p=-2,-1,0,1,2, s_{p}=12,24,13,24,23, n_{p}=36,64,36,64,64$, $\varepsilon=0.5134,1.4849,0.6520,1.0274,1.3681$ equivalent to the percentages of noise $=0.0500,0.0649,0.0494,0.0640,0.06$ The resulting reconstruction errors divided by the noises are 0.1941, 0.1240, 0.2498, 0.2361, 0.1502, demonstrating the stability of the recovery.

5.2. Three dimensions. The present framework can be easily extended to three dimensions by using the plane-wave representation

$$
\frac{e^{i \omega|\mathbf{r}|}}{|\mathbf{r}|}=\frac{i \omega}{2 \pi} \int \frac{d \alpha d \beta}{\gamma} \exp [i \omega(\alpha x+\beta y+\gamma|z|)], \quad \mathbf{r}=(x, y, z)
$$



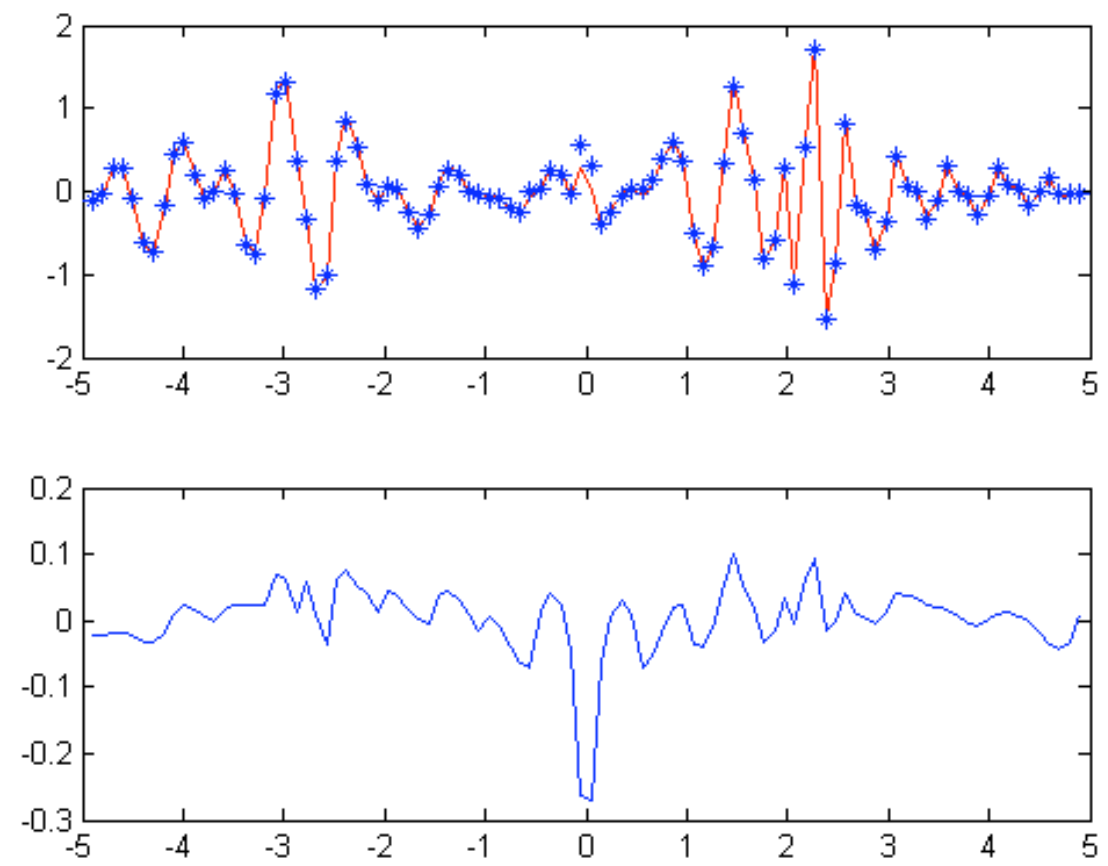

FiguRE 4. Imaging of a non-periodic target in the presence of noise: The red-solid curve (top) is the exact profile and the blue $*$ shows the reconstructed profile; the bottom plot shows the errors in reconstruction in the presence of noise.

where

$$
\begin{aligned}
& \gamma=\sqrt{1-\alpha^{2}-\beta^{2}}, \quad \alpha^{2}+\beta^{2} \leq 1 \\
& \gamma=i \sqrt{\alpha^{2}+\beta^{2}-1}, \quad \alpha^{2}+\beta^{2}>1
\end{aligned}
$$

for the Green function [1].

\section{Conclusion}

In this note, we have analyzed the problem of imaging extended targets in the perspective of Compressed Sensing which provides assurance of stable reconstruction of a target composed of sparse Fourier modes with the similar number of measurements, modulo a poly-logarithmic factor, in the presence of noise.

We have shown that the number of stably recoverable modes grows as the negative $d$-th power of the distance between the target and the sensors/source. Hence the stability of reconstructing subwavelength modes requires the distance to be less than the wavelength. On the other hand, we have also shown that the resolution limit is inversely proportional to the SNR in the high SNR limit. As a consequence, the subwavelength modes can be recovered at sufficiently high SNR as well as by placing the sensors at a subwavelength distance from the target.

However, it remains to be seen if these results about stability and resolution can be extended to the case of square-integrable targets even though our sampling scheme block-diagonalizes the corresponding scattering matrix according to each dyadic scale. 
Finally we note that the compressive imaging theory in the remote sensing regime for discrete point targets has been recently developed in $[15,16,18]$.

Acknowledgement. I thank my students Arcade Tseng (Figures 1-3) and Wenjing Liao (Figure 4) for preparing the figures. I am grateful to the anonymous referee for the helpful comments for improving the manuscript.

\section{REFERENCES}

[1] M. Born and E. Wolf, Principles of Optics, 7-th edition, Cambridge University Press, 1999.

[2] A.M. Bruckstein, D.L. Donoho and M. Elad, "From sparse solutions of systems of equations to sparse modeling of signals," SIAM Rev. 51 (2009), 34-81.

[3] E. J. Candès, "The restricted isometry property and its implications for compressed sensing," Compte Rendus de l'Academie des Sciences, Paris, Serie I. 346 (2008) 589-592.

[4] E. Candés, J. Romberg and T. Tao, "Robust undertainty principles: Exact signal reconstruction from highly incomplete frequency information," IEEE Trans. Inform. Theory $\mathbf{5 2}$ (2006), 489-509.

[5] E.J. Candès, J. Romberg and T. Tao, "Stable signal recovery from incomplete and inaccurate measurements," Commun. Pure Appl. Math. 59 (2006), 120723.

[6] E. J. Candès and T. Tao, "Decoding by linear programming," IEEE Trans. Inform. Theory $\mathbf{5 1}$ (2005), 42034215.

[7] E. J. Candès and T. Tao, "Near-optimal signal recovery from random projections: universal encoding strategies?," IEEE Trans. Inform. Theory 52 (2006), 54-6-5425.

[8] Compressive Sensing Resources. http://www.dsp.ece.rice.edu/cs/

[9] S.S. Chen, D.L. Donoho and M.A. Saunders, "Atomic decomposition by basis pursuit," SIAM Rev. 43 (2001), 129-159.

[10] D. Colton and R. Kress, Inverse Acoustic and Electromagnetic Scattering Theory. 2nd edition, Springer, 1998.

[11] I. Daubechies, Ten Lectures on Wavelets. SIAM, Philadelphia, 1992.

[12] D.L. Donoho, M. Elad and V.N. Temlyakov, "Stable recovery of sparse overcomplete representations in the presence of noise," IEEE Trans. Inform. Theory 52 (2006) 6-18.

[13] D.L. Donoho and X. Huo, "Uncertainty principle and ideal atomic decomposition," IEEE Trans. Inform. Theory 47 (2001), 2845-2862.

[14] W. Dai and O. Milenkovic, "Subspace pursuit for compressive sensing: closing the gap between performance and complexity," arXiv:0803.0811.

[15] A.C. Fannjiang, "Compressive inverse scattering I. High-frequency SIMO measurements," arXiv: 0906.5405.

[16] A.C. Fannjiang, "Compressive inverse scattering II. SISO measurements with Born scatterers," arXiv: 0908.4072.

[17] A.C. Fannjiang and K. Solna, "Broadband Resolution Analysis for Imaging with Measurement Noise ," J. Opt. Soc. Am. A 24 (2007) 1623-1632.

[18] A.C. Fannjiang, P. Yan and T. Strohmer, "Compressed remote sensing of sparse objects," arXiv: 0904.3994.

[19] D. Needell, J. A. Tropp, "CoSaMP: Iterative signal recovery from incomplete and inaccurate samples," Preprint, 2008.

[20] L. Novotny and B. Hecht, Principles of Nano-Optics, Cambridge University Press, Cambridge, 2006.

[21] H. Rauhut, "Stability results for random sampling of sparse trigonometric polynomials," preprint, 2008.

[22] M. Rudelson and R. Vershynin, "On sparse reconstruction from Fourier and Gaussian measurements," Comm. Pure Appl. Math. 111 (2008) 1025-1045.

[23] J.A. Tropp, " Just relax: convex programming methods for identifying sparse signals in noise," IEEE Trans. Inform. Theory 52 (2006), 1030-1051. "Corrigendum" IEEE Trans. Inform. Theory (2008).

E-mail address: fannjiang@math.ucdavis.edu

Department of Mathematics, University of California, Davis, CA 95616-8633 